\title{
Do clinical decision-support reminders for medical providers improve isoniazid prescription rates among HIV-positive adults?
}

Eric P. Green

Caricia Catalani

Aggrey Keny

Lameck Diero

Charity Ndwiga

Population Council

See next page for additional authors

Follow this and additional works at: https://knowledgecommons.popcouncil.org/departments_sbsr-hiv

Part of the Demography, Population, and Ecology Commons, Family, Life Course, and Society Commons, International Public Health Commons, and the Medicine and Health Commons How does access to this work benefit you? Let us know!

\section{Recommended Citation}

Green, Eric P., Caricia Catalani, Aggrey Keny, Lameck Diero, Charity Ndwiga, Dennis Israelski, and Paul Biondich. 2015. "Do clinical decision-support reminders for medical providers improve isoniazid prescription rates among HIV-positive adults?" Washington, DC: USAID I Project Search: HIVCore. 


\section{Authors}

Eric P. Green, Caricia Catalani, Aggrey Keny, Lameck Diero, Charity Ndwiga, Dennis Israelski, and Paul Biondich 


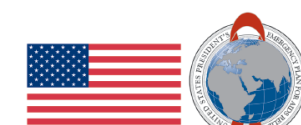
PEPFÁR

\section{DO CLINICAL DECISION- SUPPORT REMINDERS FOR MEDICAL PROVIDERS IMPROVE ISONIAZID PRESCRIPTION RATES AMONG HIV-POSITIVE ADULTS?}

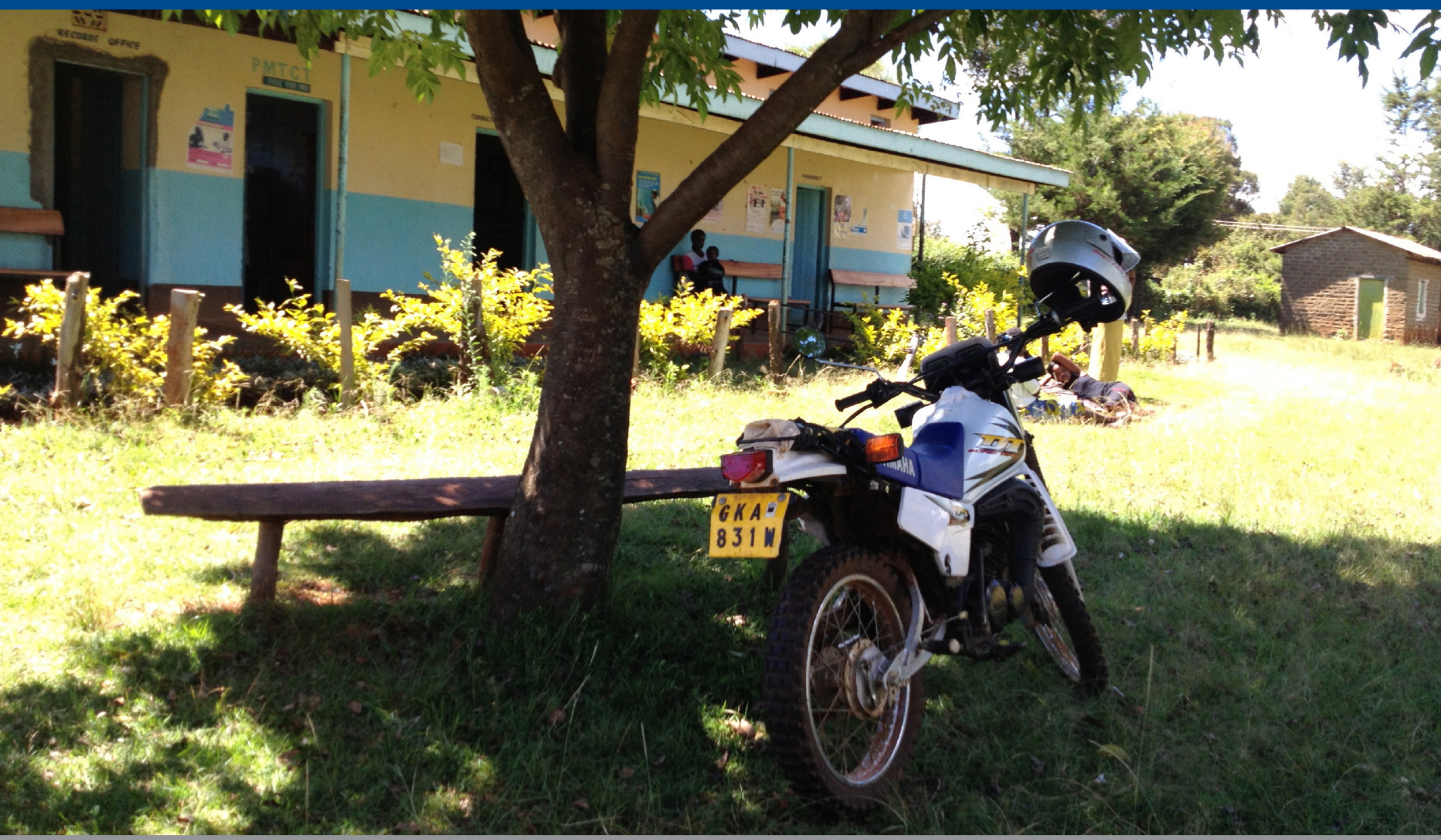

17 December 2015

This publication was produced for the United States Agency for International Development. It was prepared by Eric P. Green, Caricia Catalani, Aggrey Keny, Lameck Diero, Charity Ndwiga, Dennis Israelski, and Paul Biondich. 



\section{DO CLINICAL DECISION- SUPPORT REMINDERS FOR MEDICAL PROVIDERS IMPROVE ISONIAZID}

PRESCRIPTION RATES AMONG HIV-POSITIVE ADULTS?

ERIC P. GREEN', CARICIA CATALANI", AGGREY KENY ${ }^{3}$, LAMECK DIERO ${ }^{4}$, CHARITY NDWIGA ${ }^{5}$, DENNIS ISRAELSKI ${ }^{2}$, AND PAUL $\mathrm{BIONDICH}^{3}$

IKIJANI CONSULTING, NORTH CAROLINA, USA

2INSTEDD, SUNNYVALE, CA, USA

3REGENSTRIEF INSTITUTE, INDIANAPOLIS, IN, USA

${ }^{4}$ AMPATH, ELDORET, KENYA

${ }^{5}$ POPULATION COUNCIL, NAIROBI, KENYA

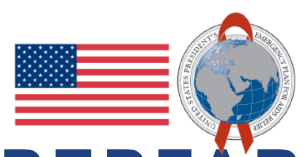

PEPFAR 


\section{ACKNOWLEDGMENTS}

The authors would like to acknowledge the contributions of Ada Yeung, Nyoman Ribeka, and the HIVCore Team at the Population Council, including Sam Kalibala, Nrupa Jani, and Deborah Weiss. We would like to particularly thank Ms. Sherry Hutchinson for her helpful guidance on all things knowledge management.
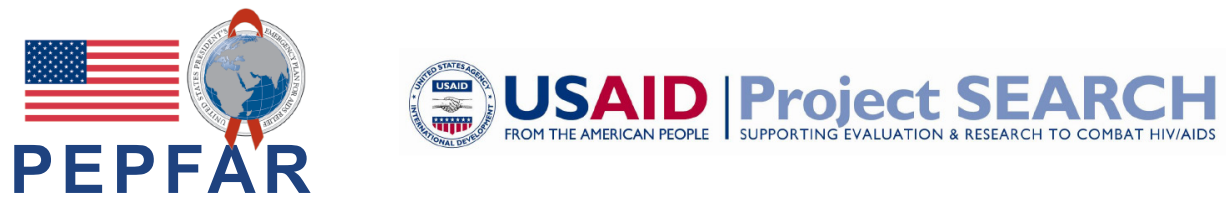

This report was made possible through support provided by the President's Emergency Plan for AIDS Relief and the U.S. Agency for International Development (USAID) via HIVCore, a Task Order funded by USAID under the Project SEARCH indefinite quantity contract (Contract No. AID-OAA-TO-11-00060).

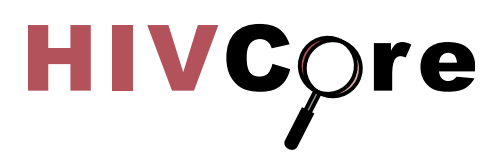

HIVCore improves the efficiency, effectiveness, scale, and quality of HIV treatment, care, and support, and prevention of mother-to-child transmission (PMTCT) programs. The Task Order is led by the Population Council in partnership with Elizabeth Glaser Pediatric AIDS Foundation, Palladium (formerly Futures Group), and the University of Washington.

Published in December 2015. @2015. The Population Council Inc.

Suggested citation: Green, Eric P., Caricia Catalani, Aggrey Keny, Lameck Diero, Charity Ndwiga, Dennis Israelski, and Paul Biondich. 2015. "Do clinical decision-support reminders for medical providers improve isoniazid prescription rates among HIV-positive adults?," HIVCore Final Report. Washington, DC: USAID | Project Search: HIVCore. 


\section{TABLE OF CONTENTS}

ACKNOWLEDGMENTS

ACRONYMS

EXECUTIVE SUMMARY

INTRODUCTION

Objective

METHODOLOGY

Design

Setting and participants

Description of the intervention

Procedures

Measurement

Statistical analysis

Ethical considerations

KEY FINDINGS

Implementation processes

Treatment effects

DISCUSSION AND LIMITATIONS

Discussion

Limitations 


\section{ACRONYMS}

$\begin{array}{ll}\text { AIDS } & \text { acquired immune deficiency syndrome } \\ \text { AMPATH } & \text { Academic Model Providing Access to Healthcare } \\ \text { AMRS } & \text { AMPATH medical record system } \\ \text { CDSS } & \text { clinical decision-support system } \\ \text { CXR } & \text { chest radiograph } \\ \text { DA } & \text { data assistant } \\ \text { EMR } & \text { electronic medical record } \\ \text { HIV } & \text { human immunodeficiency virus } \\ \text { INH } & \text { isoniazid } \\ \text { IPT } & \text { isoniazid preventive therapy } \\ \text { OpenMRS } & \text { Open Medical Records System } \\ \text { PLHIV } & \text { people living with HIV } \\ \text { SE } & \text { standard error } \\ \text { SQL } & \text { Structured Query Language } \\ \text { TB } & \text { tuberculosis }\end{array}$

USAID United States Agency for International Development 


\section{EXECUTIVE SUMMARY}

\section{INTRODUCTION}

People living with HIV (PLHIV) are at increased risk of developing active tuberculosis (TB) and dying from TB. Isoniazid (INH) preventive therapy (IPT) can prevent this, but only a small fraction of HIV-positive individuals are on IPT. Previous research suggests that clinical decision support systems (CDSS) - electronic systems that use existing patient data and established algorithms to generate alerts, reminders, or recommendations intended to aid clinical decision making - have the potential to improve provider adherence to diagnostic and treatment guidelines. Researchers conducted a cluster randomized controlled trial of a new CDSS intervention for TB screening, prevention, and treatment in a high HIV and TB prevalence setting to evaluate the effects of implementing a reminder system for medical providers to increase IPT for PLHIV. Specifically, the research objective was to determine if the intervention increases IPT prescription rates and decreases the time from initial encounter to IPT initiation.

The study was conducted as part of the HIVCore project, which is funded by the U. S. Agency for International Development (USAID) and led by the Population Council. The study team was comprised of researchers from Kijani Consulting, INSTEDD, Regenstrief Institute, AMPATH, and Population Council.

\section{METHODS}

\section{Design and setting}

The study took place in western Kenya from April to November 2014. Facilities (clusters) rather than individual providers were randomized to the study arms to prevent potential contamination and spillover effects. Twenty public medical facilities (clusters) were sorted into four strata and randomly assigned to the intervention or control arm (ratio of 1:1). Medical facilities were recruited from the AMPATH network of clinics that serves a catchment area of 3.5 million people and cares for about 150,000 HIVpositive patients, nearly half of whom are being treated for active TB.

\section{The intervention}

A new set of reminders for TB prevention and treatment based on accepted clinical algorithms was created. These reminders were designed to prompt medical providers to complete TB screenings, consider initiating anti-TB treatment or IPT, and monitor adherence to treatment based on clinical data that exist in the AMPATH medical record system (AMRS). The intervention arm received clinical summary sheets, existing reminders, and the new TB care reminders. The control arm received the summary sheets and the existing set of reminders (but no new TB care reminders). 


\section{Data analysis}

While the intervention was targeted at providers, the analysis of the outcome is based on the eligible patients. The analysis was conducted using a sample of 1,129 eligible PLHIV. To determine if there was a statistically significant difference in the proportion of INH prescriptions among IPT-eligible patients between treatment and control clusters, we used a Kruskal-Wallis test. This test was used because the data were not normally distributed. In addition, we also estimated a mixed effects logistic regression model of the relationship between assignment to treatment and patients' INH status at three months post-initial encounter. Furthermore, to evaluate whether the intervention reduced the time from initial encounter to INH prescription, we used Kaplan-Meier methods following a Cox proportional hazard regression analysis.

\section{RESULTS}

A total of 1,129 (treatment arm: 504; control arm: 625) IPT-eligible patients were included in the analysis. The reminders intervention increased the rate of INH prescriptions within three months from the initial encounter, but this effect was small and not statistically significant. The intervention also had a small positive effect on the time to prescription-the reminders led to a reduction of 4.2 days in the time from initial encounter to INH prescription; the restricted mean time in days was 174.6 (standard error $[\mathrm{SE}]=3.1)$ in the treatment group and 178.7 days $(\mathrm{SE}=3.1)$ in the control group.

\section{CONCLUSIONS}

The results suggest that the electronic reminder system may have some small effects on INH prescriptions (a 2 percent increase). In addition, it led to a reduction in the time from initial encounter to INH prescription. 


\section{INTRODUCTION}

People living with HIV (PLHIV) are 20 to 37 times more likely to develop active tuberculosis (TB) disease than those not infected with HIV, and more than one quarter of deaths among HIV-positive individuals is due to TB (World Health Organization [WHO] 2011). Active TB disease occurs at any stage of HIV infection and is often the first recognized presentation of the underlying viral infection (Sonnenberg et al. 2005; Havlir et al. 2008). As such, routinely screening for TB during HIV care creates important opportunities to diagnose and promptly treat active disease, and also to identify those without active TB disease that are eligible for isoniazid (INH) preventive therapy (IPT) (Burgess et al. 2001).

A growing body of literature suggests that IPT reduces overall TB incidence and is therefore of considerable benefit to patients and the larger community (Akolo et al 2010). For these reasons, WHO recommends routine, repeated clinical screening for active TB disease among all people living with HIV and the provision of either treatment for active disease or IPT for asymptomatic patients to mitigate the risk of developing active TB (WHO 2008). However, only a small fraction of the estimated 35 million people living with HIV initiate IPT (0.2 percent in 2009) (WHO 2010).

Barriers to IPT uptake are thought to include a lack of knowledge about treatment guidelines, fear of causing INH-resistant TB, concerns about patient adherence, and unreliable supply chains (Getahun et al. 2010; Catalani et al. 2014). No single intervention can overcome all of these barriers, but research suggests that clinical decision support systems (CDSS) —electronic systems that use existing patient data and established algorithms to generate alerts, reminders, or recommendations intended to aid clinical decision making - have the potential to improve provider adherence to diagnostic and treatment guidelines (Bright et al. 2012). While evidence of impact on patient outcomes is lacking, studies support the notion that a CDSS can improve the quality of care, even in low-resource settings (Were et al. 2013).

In an effort to expand this evidence base and promote the use of IPT among HIV-positive adults, the study team conducted a cluster randomized controlled trial of a new CDSS intervention for TB screening, prevention, and treatment in a setting of high HIV and TB prevalence in western Kenya.

The study was conducted under the HIVCore project, which is funded by the U. S. Agency for International Development (USAID) and led by the Population Council. The study team was comprised of researchers from Kijani Consulting, INSTEDD, Regenstrief Institute, AMPATH, and Population Council.

\section{OBJECTIVE}

The research objective was to evaluate the impact of implementing a reminder system for medical providers (i.e., nurses, clinical officers, medical officers, consultants) on IPT use among adults living with HIV in western Kenya.

The research question was:

Does providing medical personnel with patient-specific reminders regarding TB that are generated from a patient's electronic medical record and based on clinical algorithms for screening significantly increase IPT prescription rates and decrease the time from initial encounter to IPT initiation? 


\section{METHODOLOGY}

\section{DESIGN}

This was a cluster-randomized trial involving 20 public medical facilities (clusters) in western Kenya (Green et al. 2015). Clusters were sorted into four strata and randomly assigned via computer algorithm to the treatment or control arm with an allocation ratio of $1: 1$. The two stratifying variables were average monthly patient volume and the percentage of initial patient encounters in 2013 with chest radiograph (CXR) results recorded in the electronic medical record (EMR) database.

\section{SETTING AND PARTICIPANTS}

This study took place in the Western Region of Kenya from April to November 2014. Medical facilities were recruited from the AMPATH network of clinics that serves a catchment area of 3.5 million people. This health network cares for more than 150,000 HIV-positive patients, nearly half of whom are being treated for active TB.

Medical providers with responsibility for diagnosing $\mathrm{TB}$ and prescribing $\mathrm{TB}$ medication and IPT were the intervention targets for this study. Due to logistical challenges of randomizing providers and concerns about potential spillover within clinics, medical facilities rather than individual providers were randomized to the study arms.

To be included in the study as a "cluster," a facility had to meet the following criteria:

- Operates as an HIV care clinic

- Uses AMPATH HIV initial and return paper encounter forms (see the Appendix)

- Enters data directly into the EMR database or sends forms to central location for data entry

- Prints or receives printed paper clinical summary sheets with reminders

- Prescribes IPT

- Classified as a "mother" facility ${ }^{1}$ in the AMPATH network

At the time of randomization, there were 22 AMPATH facilities that met these criteria. The researchers excluded two facilities: (i) a large facility that operates as a regional referral hospital; and (ii) a small facility that was very new to the network.

While the intervention is targeted at the providers, the analysis is based on eligible patients. To be included in the analysis, patients had to meet the following criteria:

- HIV-positive and receiving treatment at an HIV care clinic

- Complete an adult initial encounter at a study cluster during the enrollment period

${ }^{1} \mathrm{~A}$ "mother" facility is a larger health facility that is associated with smaller 'satellite' health facilities. Providers and patients often move between a mother facility and its satellite facilities. 
- No history of TB or IPT treatment

- No evidence of active TB three months after their initial encounter

- Attend at least one additional appointment within 90 days after the initial encounter at any study facility

Patients were not "recruited" into the study, per se, as the intervention was provided to medical personnel and allocated by cluster. Instead, we ran pre-specified data queries on the EMR database to identify the cohort of eligible patients.

To be eligible for IPT, and thus eligible for our analysis, patients must have had no evidence of active TB three months after their initial encounter. The only (nearly) complete source of this evidence in the EMR database was patient report of symptoms suggestive of possible active TB (e.g., cough, night sweats). CXR data was missing for most patients; 70 percent of potential patients in our initial cohort never had a CXR conducted, despite AMPATH policy that all new HIV-positive patients receive CXRs. CXR status for another 20 percent could not be confirmed, even after case investigation.

The final eligibility criterion was that a patient must have attended at least one additional appointment within 90 days of her initial encounter so that her medical provider had the opportunity to be exposed to the intervention.

\section{DESCRIPTION OF THE INTERVENTION}

The intervention under investigation involved providing clinic-based medical care providers with patientspecific clinical reminders regarding TB that were generated from a patient's EMR (an existing digital job aide) and based on accepted clinical algorithms for TB screening and treatment. The intervention for this study, which are the TB clinical reminders, were built into this existing tool (EMR with the CDSS).

\section{Electronic medical records}

The foundation of AMPATH's existing CDSS is an electronic medical record system that is capable of tracking millions of patient encounters. The AMPATH medical record system (AMRS) is one of the world's largest implementations of the Open Medical Records System (OpenMRS) platform (Seebregts et al. 2009). The AMRS includes more than 200 million clinical observations from more than 700,000 patients. The system tracks data on test results, patient history, physical exam findings, treatment plans, adherence, and related information from clinical encounters.

AMRS is optimized for places like rural western Kenya. Medical personnel complete paper encounter forms at every patient visit, and centralized teams of data clerks enter the data into the AMRS. Pilot studies are underway to guide the transition to electronic data entry at the point of care, but currently records are digitized centrally. This makes it feasible to extend the coverage of the EMR to include the most remote facilities that do not have reliable power or access to computers or tablets.

\section{Clinical decision support}

Prior to the patient's next scheduled visit, clinic personnel print a paper clinical summary sheet that gives vital details and patient-specific care suggestions that are automatically generated by the system based on a defined set of rules. This summary sheet helps to guide providers with modest levels of training to provide high-quality, standardized care (Were et al. 2013). The summary sheets also go home with the patients. 
At the time of the trial, there were more than 60 possible clinical care reminders that could be triggered and printed on a patient's summary sheet, but there was only space to display several of the most relevant reminders for each patient. Prior to study launch, the only TB-related reminder in use was a reminder about the need to conduct and report the results of a CXR usually performed at the initial encounter to rule out active pulmonary infection.

\section{TB clinical care reminders}

As part of this study intervention, we created a new set of reminders for TB prevention and treatment based on accepted clinical algorithms (Catalani et al. 2014). These reminders (Table 1) were designed to prompt medical providers to complete TB screening, consider initiating anti-TB treatment or IPT, and monitor adherence to treatment based on clinical data that exist in the AMRS. The reminders were pretested with providers prior to launching. Providers indicated their preference for more detailed reminders over more concise reminders.

Clinical summary sheets for patients receiving care at treatment clusters included the new TB care reminders (as needed) plus the full set of existing reminders, whereas summary sheets printed at control clusters only included the existing set of reminders (no new TB care reminders).

No additional training was required for this intervention because the providers had already been using the CDSS.

\section{PROCEDURES}

We collected or accessed two types of data: information about facilities and information about patients.

\section{Facility-level data collection}

Facility-level data was collected by a team of Kenyan data assistants (DAs) under the supervision of the study manager and the AMPATH TB-HIV integration manager. DAs collected data on two facility-level indicators on a daily basis from all study facilities starting nine months prior to the start of the trial: (i) isoniazid stock (in $100 \mathrm{mg}$ and $300 \mathrm{mg}$ formulations); and (ii) summary sheet printing and distribution. A third indicator, the availability of radiography services, was documented on a weekly basis.

DAs employed various means to gather these data, including in-person facility visits and phone calls and text messages to facility staff. Remote data collection was possible when in-person visits were not because DAs spent time developing good rapport with facility staff.

DAs recorded their findings on paper and then transferred the data to a shared database at the end of each day. The database contained logical checks for data validity and alerted the DAs to potential errors.

\section{EMR database queries}

The AMPATH EMR database is a Structured Query Language (SQL) database. A team of experienced clinicians and informaticians designed and wrote SQL scripts used to query the database for relevant patient records. Query results were validated prior to analysis by checking a sample of the output against actual medical records. 
Table I TB-related clinical decision support reminders in the control and treatment groups

\begin{tabular}{|c|c|c|}
\hline CXR & Symptoms & Reminder \\
\hline \multicolumn{3}{|c|}{ CONTROL (Existing reminders) } \\
\hline Missing & NA & Patient needs baseline chest $X$-ray checked \\
\hline \multicolumn{3}{|c|}{ TREATMENT (New reminders) } \\
\hline \multirow[t]{3}{*}{ Missing } & Not recorded & $\begin{array}{l}\text { TB symptoms include chronic cough, fever, \& weight loss. Please screen patients at } \\
\text { every visit by asking all symptoms questions in short form and ordering/interpreting a } \\
\text { CXR. Most HIV+ patients should begin either anti-TB meds or IPT. }\end{array}$ \\
\hline & Symptomatic & $\begin{array}{l}\text { Your patient reported TB symptoms during the last encounter. Please order CXR to } \\
\text { determine if he or she has active TB and needs to begin lifesaving treatment. }\end{array}$ \\
\hline & Asymptomatic & $\begin{array}{l}\text { If patient still does NOT report TB symptoms, a normal CXR would suggest patient } \\
\text { is eligible for IPT, which could save his/her life. Order CXR to determine if IPT is } \\
\text { indicated or record existing results to terminate this reminder. }\end{array}$ \\
\hline \multirow[t]{3}{*}{ Normal } & Not recorded & $\begin{array}{l}\text { TB symptomatic screening results not recorded in previous encounter. Patient has } \\
\text { normal CXR. If no symptoms, should consider initiating IPT. IPT saves lives. }\end{array}$ \\
\hline & Symptomatic & $\begin{array}{l}\text { Your patient reported TB symptoms during the last encounter. CXR results were } \\
\text { normal. Please order further tests such as Sputum microscopy to rule out presence of } \\
\text { TB in your patient. }\end{array}$ \\
\hline & Asymptomatic & $\begin{array}{l}\text { Test results do NOT suggest active TB. If patient still does NOT report TB symptoms, } \\
\text { consider initiating IPT. IPT is effective and could save his/her life. }\end{array}$ \\
\hline \multirow[t]{3}{*}{ Abnormal } & Not recorded & $\begin{array}{l}\text { TB symptomatic screening results not recorded in previous encounter. Patient has } \\
\text { Abnormal CXR. If patient has symptoms, should consider initiating TB treatment. TB } \\
\text { treatment saves lives. }\end{array}$ \\
\hline & Symptomatic & $\begin{array}{l}\text { Your patient may have TB. They reported TB symptoms during the last visit and had an } \\
\text { abnormal CXR. Order sputum test to determine if she/he should start life-saving TB } \\
\text { treatment today. }\end{array}$ \\
\hline & Asymptomatic & $\begin{array}{l}\text { Your patient reported no TB symptoms during the last encounter, however CXR } \\
\text { results were abnormal. Please order further tests such as Sputum microscopy to rule } \\
\text { out presence of TB in your patient. }\end{array}$ \\
\hline
\end{tabular}

\section{MEASUREMENT}

INH was considered "prescribed" for a patient when there was an entry in the EMR database indicating that a medical provider prescribed INH (binary yes/no outcome). The denominator for the calculation of the cluster-level prescription rate was the number of IPT-eligible patients who met study eligibility. Thus 
the prescription rate was calculated as the proportion of IPT-eligible patients who were prescribed INH within three months of his or her initial encounter (first visit).

INH prescription time lag was determined by calculating the number of days from a patient's initial encounter to INH prescription.

We also tracked cluster treatment compliance, crossover of providers and patients, and process indicators on INH stock and the availability of radiography services.

\section{Process indicators}

A. Cluster-level treatment compliance: Clusters were randomly assigned to receive the new TB reminders + the existing CDSS reminders (treatment) or just the existing CDSS reminders (control). Thus to be compliant, clusters needed to print correct summary sheets, deliver the sheets to all cluster facilities if printing was not done on-site, and ensure that the sheets were inserted into patients' medical files before encounters.

A1. Generate correct summary sheets: A "correct" summary sheet was one in which TB reminders were either present or absent depending on the cluster treatment status and details in a patient's electronic medical record. Prior to study launch, we tested all reminders against hundreds of patient records to ensure accuracy. As part of ongoing quality assurance, team members periodically checked printed reminders and system logs for accuracy.

A2. Printing, delivery, and filing of summary sheets: Once generated, facilities had to print the paper summary sheet prior to the patient's next encounter. Some facilities printed on-site; others had sheets delivered from the closest facility with printing capability. Some of the printing facilities printed summary sheets "on-demand" as patients arrived; others printed in advance. Facilities that had summary sheets delivered and those that printed in advance also had to place the papers in patient folders prior to each encounter.

For reminders to be effective, the summary sheets containing the reminders had to be inserted into a patient's file prior to the encounter with the medical provider. There was no system in place to record that this happened for every patient encounter. Study investigators did, however, track facility-level compliance through random spot checks. The team defined facility-level compliance as the percentage of patient encounters that occurred with a recent summary sheet present at the time of the encounter.

Facility-level compliance data was collected through the team of DAs. DAs were encouraged to visit facilities in person as often as possible, but they were permitted to accept remote reports when not feasible to visit. When DAs visited facilities, they observed the following: (i) summary sheets printed in-advance of scheduled patient encounters (printed the day before or earlier, sometimes off-site and delivered) and/ or (ii) summary sheets printed on-demand for scheduled and unscheduled patient encounters.

For (i), the denominator was defined as the number of files checked and the numerator was defined as the number of files checked with recent summary sheets present. For (ii), the denominator was defined as the number of clinical encounters that occurred during the observation period and the numerator was the number of clinical encounters during this period in which a summary sheet was printed on-demand. 
When DAs accepted remote reports, they documented the following from facility contacts: (a) the number of return encounters (denominator) and the number of return encounters that occurred with a recent summary sheet present at the time of the encounter (numerator); or (b) a minimal report of the facility's summary sheet printing/distribution status (operational or not operational).

When daily numerator and denominator counts were provided, we calculated the facility-level percentage of patient encounters that occurred with a recent summary sheet present at the time of the encounter. For observations in which the DA was only able to indicate a printing/distribution status of "operational," we imputed the facility mean proportion for that observation.

B. Medical provider treatment compliance: Medical providers could also be a source of cluster noncompliance. For instance, a medical provider who crossed over between treatment and control clusters would be non-compliant with her cluster's assigned treatment status. The location of all encounters was recorded on every encounter form, allowing us to track potential crossover.

C. Patient treatment compliance: The reminders treatment was administered at the cluster level, but our primary outcome (INH prescription) was observed at the patient-level. Like medical providers, patients could be non-compliant if they crossed over between treatment and control clusters to receive care. However, an analysis of 2012 AMPATH data suggested that patient encounters at multiple sites are rare. When multiple-site treatment does occur, it is usually within a single cluster of mother and satellite sites, thus avoiding the risk of contamination. We tracked encounter locations through the EMR database.

D. Isoniazid stock: This process indicator was defined as the count of $100 \mathrm{mg}$ and $300 \mathrm{mg}$ INH tablets available at the end of the day of data collection (reported in $100 \mathrm{mg}$ units). DAs were encouraged to visit facilities in-person as often as possible to obtain the count of $100 \mathrm{mg}$ and $300 \mathrm{mg}$ tablets from pharmacies. When in-person visits were not possible, DAs were permitted to record facility-reported stock reported over the phone or via SMS.

In cases where DAs could not visit the facility and the facility contact could not estimate the stock, the DA recorded a binary indicator of stock (present or absent). When this happened, we imputed the facility mean stock for observations where the DA was only able to indicate "present." The final indicator was facility Isoniazid stock in $100 \mathrm{mg}$ tablets.

E. Availability of radiography services: This weekly process indicator was defined as the number of work days $(\mathrm{M}-\mathrm{F})$ in which a facility's radiography services were operational.

\section{STATISTICAL ANALYSIS}

We designed this study to detect a minimum shift of 8 percentage points in the INH prescription rate, from 15 percent to 23 percent using a one-tailed test. This assumed a power of 0.80 , alpha of 0.05 , 20 clusters equally allocated to treatment and control, 50 patients per cluster, and a between-cluster coefficient of variation of 0.25 .

To determine if there was a statistically significant difference in the proportion of INH prescriptions among IPT-eligible patients between treatment and control clusters, we used a Kruskal-Wallis test, a nonparametric alternative to an unpaired t-test of group mean differences because the results of a ShapiroWilk normality test suggested that the distribution of cluster proportions was not normal. The Kruskal- 
Wallis test rank orders the rate of INH prescriptions, and then compares which group has higher ranked rates, thus it is not dependent on the distribution of the raw data, as all data are converted into rank order.

In addition to this analysis of cluster-level summary data, we also estimated a mixed effects logistic regression model of the relationship between assignment to treatment and patients' INH status at three months post-initial encounter; intercepts for clusters were entered as random effects. Both analyses were intent-to-treat.

As an alternative to evaluating patients' INH status after a defined window of time (i.e., three months post-initial encounter), we also fit a Cox proportional hazards model that regressed "survival" times on clinic assignment to treatment (with controls for patient age and gender). To determine whether the intervention reduced the time from initial encounter to INH prescription, we used Kaplan-Meier methods to calculate the proportion of eligible patients receiving INH prescriptions within three months of their initial encounters and used the log rank test to test the null hypothesis that there is no difference between the survival curves.

\section{ETHICAL CONSIDERATIONS}

This protocol was reviewed and approved by the Moi University School of Medicine/Moi Teaching and Referral Hospital Institutional Research and Ethics Committee (IREC; 0001055), the Indiana University Institutional Review Board (1307011750), and the Lifespan Research Protection Office (0000396, 00004624). The approving ethics committees did not require individual patient consent given the nature of the intervention. 


\section{KEY FINDINGS}

All 20 clusters randomized to the treatment and control conditions participated in the study and were included in the analysis (Figure 1). Between 11 April 2014 and 11 August 2014, 2,211 HIV-positive adult patients had initial encounters at one of the 20 study clusters. One thousand forty-two were excluded from further analysis because they had a history of IPT or TB, or because they had evidence of active TB. An additional 40 patients were excluded because they were determined to be ineligible for IPT at the three month post-initial encounter snapshot. Thus, a total of 1,129 patients were included in the analysis sample.

\section{Figure I Consort diagram}

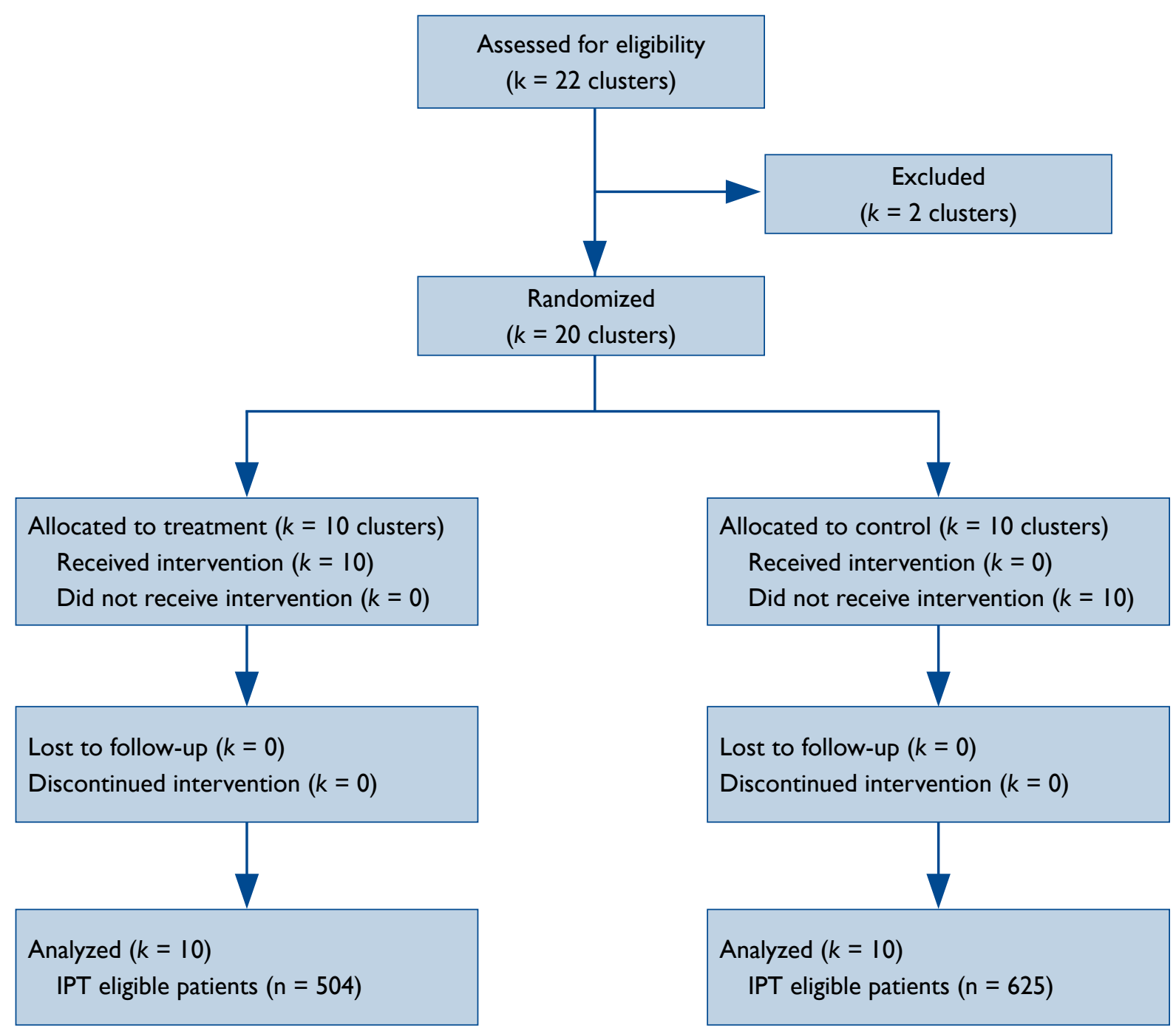




\section{IMPLEMENTATION PROCESSES}

\section{Facility-level compliance}

When the trial began, mean uptime (CDSS summary sheet system was functional) in the two groups was converging to between 60 and 65 percent. This means that, on average, the CDSS summary sheet system was non-functional 35 to 40 percent of the time around the start of the trial. When the system was not functioning, clinicians at control facilities did not view the standard clinical summary sheet and clinicians at treatment facilities did not view the new clinical summary sheet that included the TB-related reminders. Since the intervention could not be delivered to facilities assigned to treatment when the system was not functioning, the effect of downtime was to attenuate any treatment effect because the contrast between treatment and control facilities was reduced.

\section{Use of TB reminders}

Another important process indicator is the content of the summary sheets. On summary sheets, the average number of reminders was 4.6 non-TB (existing) reminders and 0.8 new TB reminders. In 74.5 percent of summary sheets displaying a new $\mathrm{TB}$ reminder, the reminder that appeared told providers that the patient needed a CXR to determine IPT eligibility:

If patient still does NOT report TB symptoms, a normal CXR would suggest patient is eligible for IPT, which could save [his/her depending on gender] life. Order CXR to determine if IPT is indicated or record existing results to terminate this reminder.

This reminder appeared frequently because most patients did not report symptoms of possible active TB at their last encounter and were missing CXR results in the EMR database. ${ }^{2}$ The text of this reminder is similar to the existing reminder displayed for patients missing CXR results at control clusters: "Patient needs baseline Chest X-ray checked". Thus, summary sheets looked very similar in terms of reminder content across treatment and control clusters. This should have reduced the treatment-control contrast and attenuated any possible treatment effect.

Other new TB reminders fired in less than 5 percent of cases. The new reminder urging the provider to consider IPT (required evidence of normal CXR results and no possible TB symptoms) never fired. Given the lack of CXR results in the AMRS, this reminder should not have fired frequently, but it should have fired occasionally. This points to the possibility of a bug in the summary sheet module that governs the generation of reminders.

\section{TREATMENT EFFECTS}

\section{INH prescriptions}

The reminders intervention increased the rate of INH prescriptions, but this effect was small and not statistically significant. The data show that the overall proportion of INH prescriptions by treatment

${ }^{2}$ Reasons for missing CXR results included the fact that not all study sites had X-ray machines and even those that had these machines were experiencing challenges such as lack of staff, lack of chemicals, and power outages. As a consequence, if a patient is receiving care at a facility that does not have a CXR facility, or if the CXR machine is not operational, the patient needed to travel to the nearest facility that offers free CXRs resulting in some patients never obtaining the CXR. Sometimes CXRs were performed but the patients did not bring the films to the provider. 
and control arms was $96 / 504(0.19)$ and 103/625 (0.16), respectively (Table 2). However, the mean of cluster proportions is the preferred calculation because it takes varying cluster size into account (Hayes and Moulton 2009). At the cluster level, 24.0 percent of IPT-eligible patients at treatment clusters were prescribed INH (bootstrapped 95\% CI 13.8-35.8) compared to 21.6 of IPT-eligible patients in control clusters (bootstrapped 95\% CI 10.9-33.2), a difference of 2.4 percentage points. A t-test of the cluster proportions for INH prescriptions gives a t-statistic of $-0.3(18 \mathrm{df}, \mathrm{p}=0.61)$, suggesting that the null hypothesis of no difference cannot be rejected. As with the t-test, Kruskal-Wallis test of differences suggests that the null hypothesis of no difference-this time in mean ranks—cannot be rejected $(H(1)=0.1, p=0.71)$.

Table 2 Results of cluster-level analysis

\begin{tabular}{|lcc|}
\hline Characteristics & Treatment & Control \\
\hline Clusters randomized & 10 & 10 \\
Clusters analyzed & 10 & 10 \\
Initial encounters & 836 & 1,375 \\
Study eligible & 522 & 647 \\
IPT eligible & 504 & 625 \\
Mean number IPT eligible patients by facility (SD) & $50.4(29.2)$ & $62.5(82.3)$ \\
Percent female & 66.1 & 66.7 \\
Mean age (SD) & $37.1(10.8)$ & $37.5(10.4)$ \\
Proportion prescribed INH within 90 days (\%) & $96 / 504(0.19)$ & $103 / 625(0.16)$ \\
Mean of cluster proportions* & 0.24 & 0.22 \\
\hline
\end{tabular}

$*_{p}=0.57$

We come to the same conclusion by estimating a mixed effects logistic regression of the relationship between assignment to treatment and INH status at 3-months post-initial encounter. The odds of being prescribed INH were slightly higher among patients at treatment clusters compared to patients at control clusters $(\mathrm{OR}=1.11, \mathrm{SE}=1.57)$. The bootstrapped 90 percent confidence interval, however, is wide and includes 1.0 (0.5 to 3.1), which casts doubt upon the direction and magnitude of the effect. Yet it is also the case that 1.9 times as many bootstrapped resamples are greater than zero, suggesting that the effect is likely to be positive.

\section{Time from initial encounter to INH prescription}

Holding age, gender, and strata constant, we find that clinic assignment to treatment had essentially no impact on the hazard of time to treatment with INH prescription: 0.92 ( -0.08 percent) on average, $p=0.76$. When not controlling for gender, age, and strata, the estimated hazard of time to INH prescription increases by 1.15 times if the patient received their INH prescription from a treatment facility, but this effect is not statistically significant $(p=0.76)$. This unadjusted model suggests a small reduction in the time from initial encounter to INH prescription of 4.2 days; the restricted mean time in days was $174.6(\mathrm{SE}=3.1)$ in the treatment group and 178.7 days $(\mathrm{SE}=3.1)$ in the control group. 


\section{DISCUSSION AND LIMITATIONS}

\section{DISCUSSION}

To our knowledge, this is the first study to evaluate the use of clinical decision support reminders to increase the uptake of IPT in a TB/HIV co-morbid population. We conducted this trial in a unique setting characterized by high rates of poverty, HIV, and TB, but also one in which the health system has an established EMR and CDSS. Therefore, we were able to randomize medical facilities to receive a set of new TB-related reminders and estimate the impact of just these reminders on IPT uptake, as opposed to estimating the impact of implementing an entire EMR/decision support system with new TB-related reminders.

This study examined the use of clinical decision support reminders to improve rates of initiation of preventive treatment in a TB/HIV co-morbid population living in a TB endemic area. The reminders intervention increased the rate of INH prescriptions within three months from the initial encounter, but this effect was small and not statistically significant. The intervention also had a small positive effect on the time to prescription - the reminders led to a small reduction in the time from initial encounter to INH prescription of 4.2 days-but this finding was not robust due to the use of additional controls. A number of factors could have influenced these results including the reminders' system, clinician behavior, and health system challenges.

We found that, on average, the CDSS was non-functional more than a third of the time when the trial launched. This means that in more than one out of three patient encounters, medical providers did not have access to a recent clinical summary sheet that might have contained TB reminders (i.e., the intervention). Additionally, we learned that three out of every four times a new TB reminder was displayed on the summary sheet, it was the specific reminder that encouraged providers to order a CXR because so many new patients were missing a CXR result. Since there was already a similar (though more simplistic) "order CXR" reminder in the system and therefore available to the control facilities, this meant that our intervention was further diluted. The effect of both was likely an attenuation of the treatment effect.

\section{LIMITATIONS}

A key health systems challenge was the AMPATH guidelines requiring a "normal" CXR before a patient is put on IPT because CXR availability was erratic. It could be argued that the CXR challenges could have an equal effect in both arms. However, it should be noted that the vast majority ( 74.5 percent) of the TB reminders generated by the CDSS indicated that the patient did not have a CXR result on file. Incidentally, the text of the new CXR reminder in the intervention arm was similar to the existing reminder displayed for patients missing CXR results at control clusters: "Patient needs baseline Chest 
X-ray checked". This should have reduced the treatment-control contrast and attenuated any possible treatment effect.

There were several additional factors occurring external but simultaneously to this pragmatic trial that may have dampened out increasing uptake by the clinical reminders overall. AMPATH initiated an IPT program when it opened its doors in 2000 to treat HIV; no other site in the country was performing IPT in HIV populations. Thus, the CXR requirement was included at that time post discussions with the National Programs due to the concern of treating active TB in this population inadvertently with IPT. By the time of this trial, the national HIV and TB programs had initiated IPT programs countrywide that did not require a prior CXR (in line with WHO guidelines). Thus, clinicians were potentially receiving mixed messages-one from the national programs not to use chest radiography and one from AMPATH to do so. Clearly, given that the majority of the reminders concerned chest radiography, these conflicting directives may have led providers to ignore the AMPATH reminders regarding radiography. Finally, there were simultaneously structural programs within AMPATH as well as within the national program, including decentralization from a national program to a county program, which may have led clinicians to be unclear as to acceptance and adherence to proposed guidelines. While this may not have been the most representative or relevant site in which to conduct this research, it would have been challenging to find a better suitable setting for this particular intervention.

It is also possible that overall rates of INH prescription remained low due to provider-specific barriers, including a lack of knowledge about treatment guidelines, fear of causing Isoniazid resistant TB, concerns about patient adherence, and unreliable supply chains (Getahun et al. 2010; Catalani et al. 2014). Similar factors were observed in our preliminary study-providers were reluctant to prescribe INH if they were not sure that the stock of INH available in the pharmacy was sufficient to cover the nine months of IPT required by AMPATH. In the years immediately prior to the launch of the trial, AMPATH was perpetually experiencing INH stock outs. During the trial period, however, INH stocks were sufficient. AMPATH organized a "re-training" for providers just prior to the launch of the trial to clarify guidelines and make assurances about the supply chain, but anecdotally these barriers remained. 


\section{CONCLUSIONS AND RECOMMENDATIONS}

The results suggest that there may have been a very small, positive effect on INH prescriptions (a shift in the prevalence of less than 2 percentage points). Because the marginal cost of adding additional reminders, as we did in this study, is essentially zero, even a 2 percentage point increase in the INH prescription rate is valuable since it could mean starting thousands of HIV-positive patients on potentially life-saving prophylaxis.

Resolving the challenges in the CDSS, the health system, and the provider behaviors demonstrated by this study and potentially realizing larger intervention effects would require system-wide policy changes and improvements to basic CDSS functioning. This would likely come at considerable cost, including costs associated with staff retraining (e.g., changing treatment guidelines to eliminate the requirement for a clean CXR result) and investments in infrastructure (e.g., power, printing supplies, wireless network expansion) and human resources. Moving to an all-digital EMR platform-from point-of-care electronic data entry and reminders - could help, but experience in high-income settings suggests that this is not certain (Black et al. 2011).

Given the high costs of TB treatment and the morbidity and mortality burden associated with co-morbid HIV and TB, however, these upgrades could be cost-effective, especially considering that the CDSS supports a range of decision support interventions, not just TB. We know that this particular systemevaluated in the same context, albeit at one facility-does improve pediatric HIV care by encouraging providers to complete overdue clinical tasks (Were at al. 2013). Based on the implementation challenges of this study, the study team makes the following specific recommendations:

- Further study of the impact of a CDSS-generated reminder system would benefit from prior assurance of consistent performance of the system in low resource settings to minimize down time of the system.

- There is need for increased advocacy for IPT among decision-makers and funders to ensure a consistent supply of INH. ${ }^{3}$

- Given the lack of CXR in low resource settings AMPATH should review and consider removing the CXR requirement for IPT. This could reduce missed opportunities to initiate life-saving IPT when patients cannot obtain CXRs.

- It is important to have clear guidelines for IPT and promote them among all health providers in the system as well as train them in the required skills. In addition, there is a need to synchronize AMPATH guidelines on duration of IPT and requirement of CXR.

${ }^{3}$ AMPATH has implemented this recommendation. 


\section{REFERENCES}

Akanbi, Maxwell O. et al. 2012. "Use of electronic health records in sub-Saharan Africa: Progress and challenges," Journal of Medicine in the Tropics 14(1):1-6.

Akolo, Christopher et al. 2010. "Treatment of latent tuberculosis infection in HIV infected persons," Cochrane Database Syst Rev, 1.

Black, Ashly D. et al. 2011. "The impact of eHealth on the quality and safety of health care: A systematic overview," PLoS Med 8(1):e1000387.

Bright, Tiffani J. et al. 2012. "Effect of clinical decision-support systems: A systematic review," Annals of Internal Medicine 157(1): 29-43.

Burgess, Amelia L. et al. 2001. "Integration of tuberculosis screening at an HIV voluntary counselling and testing centre in Haiti," Aids 15(14): 1875-1879.

Catalani, C. et al. 2014. "A clinical decision support system for integrating tuberculosis and HIV care in Kenya: A human-centered design approach," Plos One 9(8):e103205.

Getahun, Haileyesus et al. 2010. "Implementation of isoniazid preventive therapy for people living with HIV worldwide: barriers and solutions," Aids 24(Suppl 5):S57-65.

Green, Eric P. et al. 2015. "Do clinical decision-support reminders for medical providers improve isoniazid preventive therapy prescription rates among HIV-positive adults?: study protocol for a randomized controlled trial," Trials 16(1):141.

Havlir, Diane V. et al. 2008. "Opportunities and challenges for HIV care in overlapping HIV and TB epidemics," JAMA: the Journal of the American Medical Association 300(4):423-430.

Seebregts, Christopher J. et al. 2009. "The OpenMRS implementers network," International Journal of Medical Informatics 78(11):711-720.

Sonnenberg, Pam et al. 2005. "How soon after infection with HIV does the risk of tuberculosis start to increase? A retrospective cohort study in South African gold miners," Journal of Infectious Diseases 191(2):150-158.

Were, Martin C. et al. 2013. "Computer-generated reminders and quality of pediatric HIV care in a resourcelimited setting," Pediatrics 131(3): e789-e796.

WHO. 2008. "WHO three 'I's meeting: intensified case finding, isoniazid preventive therapy and TB infection control for people living with HIV," WHO technical report. Geneva: WHO.

WHO. 2010. “Global tuberculosis report 2010,” WHO technical report. Geneva: WHO.

WHO. 2011. "Guidelines for intensified tuberculosis case-finding and isoniazid preventive therapy for people living with HIV in resource-constrained settings," Technical report. Geneva: WHO. 
U.S. Agency for International Development I 300 Pennsylvania Avenue, NW

Washington, DC 20523

Tel: (202) 7I2-0000

Fax: (202) 216-3524

www.usaid.gov 